comparison of the data obtained, the adverse events reported for each drug were normalized using the number of treatments for the same period. The reporting odds ratio $(\mathrm{ROR})$ and its $95 \%$ confidence intervals $(\mathrm{Cl})$ were calculated regarding the different categories of adverse events. The incidence of serious adverse events, serious infections, withdrawals due to adverse events and deaths were also calculated.

Results: The EudraVigilance database contains 851882 adverse events reported for IFX, ETN, and ADA. During this period, the different TNF antagonists have shown almost the same safety profile. The reported adverse events were classified by systems organ class (SOC) and the most frequent were administration site conditions $(28.8 \%)$ and infections and infestations $(11.2 \%)$, Safety was not statistically different. The comparison between IFX originator and its biosimilar did not show statistically significant differences in safety (ROR $1.08[0.80,1.46])$ during the initial 3 -years after launch for both drugs. However, a small nonsignificant increase in immune reactions during administration was reported for IFX-biosimilar, which might reflect increasing attention for this class of drugs.

Conclusions: The comparison of reference IFX and IFX-biosimilar did not demonstrate statistically significant differences in safety. This pharmacovigilance study provides the first analysis of TNF antagonists from the EudraVigilance database and offers a framework for safety comparison between originators and biosimilar TNF antagonists.

Acknowledgements: We would like to acknowledge Eudravigilance for providing access to the database.

Disclosure of Interest: None declared

DOI: 10.1136/annrheumdis-2017-eular.5884

\section{SAT0047 PREDICTING MAINTENANCE OF RESPONSE BASED ON DISEASE CHARACTERISTICS AND EARLY CLINICAL RESPONSE IN RHEUMATOID ARTHRITIS PATIENTS UPON WITHDRAWAL OF ADALIMUMAB}

J.S. Smolen ${ }^{1}$, P. Emery ${ }^{2}$, H. Zhang ${ }^{3}$, X. Wang ${ }^{3}$, J. Suboticki ${ }^{3}$, I. Sainsbury ${ }^{3}$, A. Kavanaugh ${ }^{4}$. ${ }^{1}$ Medical Univ of Vienna, Vienna, Austria; ${ }^{2}$ Leeds Inst of Rheumatic \& Musculoskeletal Medicine, Leeds, United Kingdom; ${ }^{3}$ AbbVie, N Chicago, IL; ${ }^{4}$ Univ of California, San Diego, CA, United States

Background: Some patients (pts) with rheumatoid arthritis (RA) achieve low disease activity (LDA) after treatment with adalimumab (ADA) plus methotrexate (MTX) and can maintain LDA after ADA withdrawal ${ }^{1}$. However, others experience a flare in disease activity. The factors associated with loss or maintenance of response are not understood.

Objectives: To identify pt disease characteristics and early clinical responses, which predict maintenance of LDA upon ADA withdrawal in individual RA pts.

Methods: Data from the OPTIMA trial were used in this post hoc analysis. In period $1(\mathrm{P} 1)$, pts were treated for 26 weeks (wks) with ADA+MTX or placebo (PBO) +MTX. Pts on ADA+MTX who achieved DAS28-CRP $<3.2$ at wks 22 and 26 (responders) were randomized to ADA withdrawal, or ADA+MTX continuation up to Wk 78. Responders to PBO+MTX in P1 continued on PBO+MTX up to Wk 78 (PBO continuation). Data from the ADA withdrawal arm were used to predict LDA at Wk 78 by DAS28-CRP $(\leq 3.2)$ or SDAI $(\leq 11)$. Potential factors including baseline (BL) disease characteristics and Wk 26 responses, including DAS28CRP, SDAl, ACR score components, modified total sharp score (mTSS) and joint space narrowing score (JSN), were screened by the LASSO method ${ }^{2}$, which performs variable selection by penalizing unduly complicated models, with/without incorporating the speed of DAS28-CRP or SDAI response as an individual predictor. Logistic regression on the LASSO-selected factors yielded coefficients used to derive individual scoring equations and prediction scores for Wk 78 outcomes (fig footnote). Prediction score cutoffs were established by the regression tree method $^{3}$. The results were validated in data from the PBO continuation arm. Results: For the prediction of DAS28-CRP LDA at Wk 78, BL physician global assessment (PhGA) and health-assessment questionnaire-disability index (HAQ-DI), and Wk 26 DAS28-CRP, HAQ-DI, JSN and CRP were selected by LASSO, and used to calculate the prediction score. Including speed of response did not affect the predictors chosen. Out of 9 pts predicted not to have DAS28-CRP LDA at Wk 78,0 had LDA (NPV=100\%) (fig 1). Out of 66 pts predicted to have DAS28-CRP LDA at Wk 78, 63 predictions were correct (PPV=96.5\%). Results were comparable for most cutoff categories in the validation arm $(\mathrm{PPV}=82 \%)$; however, no pts were predicted to have a non-response at Wk 78 . For the prediction of SDAI LDA at Wk 78, the NPV was $86 \%$ (1/7 predictions incorrect); PPV was $95 \%$ (39/41 predictions correct); in the validation arm, the PPV was $82 \%$. Conclusions: DAS28-CRP LDA at 78 wks could be individually predicted for up to $63 \%$ pts in OPTIMA who withdrew ADA/continued PBO+MTX with $96.5 \%$ accuracy, based on demographics and clinical outcomes at 6 months. This instrument could help identify pts who may be able to maintain LDA upon ADA withdrawal.

References:

[1] Smolen et al, 2013. Lancet;383.

[2] Tibshirani, R, 1996. J Royal and Stat Society; 58.

[3] Breiman L., et al, 1984. Classification and Regression Trees. Wadsworth.

Acknowledgements: AbbVie: study sponsor, contributed to design, data collection, analysis, interpretation; and writing, reviewing, approval of final version. Medical writing: Naina Barretto of AbbVie.

Disclosure of Interest: J. Smolen Grant/research support from: AbbVie, Consul-

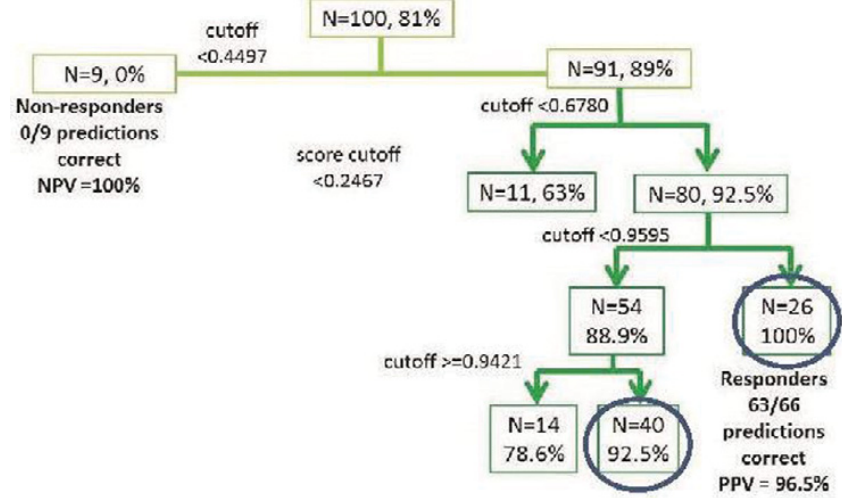

Figure 1: Regression tree to predict Week 78 DAS28-CRP LDA. The percentages of patients with an LDA response at Week 78 are indicated in the boxes. The encircled boxes indicate the patients predicted to have LDA at Week 78 .

Individual scoring equation for reaching Week 78 DAS28_LDA: Logit $=6.717-0.212^{*} \mathrm{HAQ}-0.039$, PhGA - 0.004 "DAS28_WK26 - 1.393*HAQ_WK26 - 0.027*JSN - 0.292*CRP_WK26

For reaching Week 78 SDAI LDA: Logit $=4.668-0.028$ *PhGA $-0.161^{*} \mathrm{CRP}$ W26 -

$1.411^{*}$ RoW_indicator; RoW = rest of the world, including Argentina, Australia, New Zealand, South Africa Individual prediction s core: $\frac{\exp (\log i t)}{1+\exp (\log i t)}$.

tant for: AbbVie, P. Emery Grant/research support from: from Pfizer, MSD, AbbVie Inc., BMS, UCB, Roche, Novartis, Samsung, Sandoz and Lilly., Consultant for: from Pfizer, MSD, AbbVie Inc., BMS, UCB, Roche, Novartis, Samsung, Sandoz and Lilly., H. Zhang Employee of: AbbVie, X. Wang Employee of: AbbVie, J. Suboticki Employee of: AbbVie, I. Sainsbury Employee of: AbbVie, A. Kavanaugh Grant/research support from: AbbVie Inc., Amgen, Astra-Zeneca, BMS, Celgene, Centocor-Janssen, Pfizer, Roche, and UCB., Consultant for: AbbVie Inc., Amgen, Astra-Zeneca, BMS, Celgene, Centocor-Janssen, Pfizer, Roche, and UCB. DOI: 10.1136/annrheumdis-2017-eular.2503

\section{SAT0048 THE PATTERN OF ACPA REACTIVITIES IN ANTI-CCP POSITIVE INDIVIDUALS WITH NON-SPECIFIC MUSCULOSKELETAL SYMPTOMS AT RISK OF DEVELOPING RA}

A. Hensvold ${ }^{1}$, Y. Kisten ${ }^{1,1}$, V. Joshua ${ }^{1}$, M. Hansson ${ }^{1}$, L. Mathsson-Alm ${ }^{2}$, A. Karlsson ${ }^{1}$, H. Rezaei ${ }^{1}$, E. af Klint ${ }^{1}$, G. Fei ${ }^{1}$, A. Catrina ${ }^{1},{ }^{1}$ Karolinska University Hospital and Karolinska Institutet, Stockholm; ${ }^{2}$ ThermoFisher Scientific, Uppsala, Sweden

Background: There is today a paucity of prospective studies to describe the natural longitudinal history of anti-ccp positive individuals developing RA or not developing RA. Further no study to investigate the detailed ACPA reactivities in such a setting is currently available.

Methods: Individuals at risk of developing RA were included in a cohort at Karolinska University Hospital, Stockholm. Examinations of peripheral joints was repeated at one year follow-up visit or at any time the patients experienced worsening of their symptoms. Peripheral blood samples were available at inclusion $(n=70)$. Serum was run on a microarray based on the ImmuoCAP ISAC system testing for ACPA reactivities toward 13 different citrullinated peptides (fillagrin, fibrinogen, alpha-enolase, vimentin, histone) (1).

Results: Individuals referred from primary care with musculoskeletal complaints and positive anti-ccp test were systematically investigated as part of routine care at our rheumatology clinic. Individuals lacking self-reported history of suspect arthritis, clinical arthritis according to rheumatologist and signs of synovitis on ultrasound examination were included in a clinical Risk-RA program with life-style coaching and personalized information on the risk of developing RA. Seventy individuals, with a mean age of 48 years (SD 15) and $86 \%$ females, were included in the program. Twenty (29\%) individuals developed arthritis during a medium follow up time of 7 months (range 1-25 months).

Number of ACPA reactivities at baseline was significant higher among those developing (in mean 6 reactivities) as compared to those not developing arthritis (in mean 4 reactivities). A increased proportion of individuals were showing reactivity towards citrullinated (cit) vimentin (vim) 60-75, fibrinogen (fib) 573 and enolase (eno) (CEP-1) among those developing arthritis (80\% for anti-cit-vim $45 \%$ for anti-cit-fib and $60 \%$ for anti-cit eno) as compared to those not developing arthritis $(41 \%$ for anti-cit-vim, $30 \%$ for anti-cit-fib and $52 \%$ for anti-cit-eno). Increased level of anti-cit-vim and anti-cit-eno antibodies was also observed at inclusion for those individuals developing arthritis as compared to those not developing arthritis.

Conclusions: We describe here the pattern of ACPA reactivities in antiCCP positive individuals with non-specific musculoskeletal symptoms at risk of developing RA and without clinical and ultrasonograph signs of synovitis and report that $30 \%$ of these patients will develop arthritis during a short follow-up. Number, frequency and titers of specific ACPA reactivities appear to be enriched already at inclusion among those patients that developed arthritis during follow-up. References:

[1] Hansson et al Arthritis Res Ther 2012. 